\title{
Propiedades psicométricas del Cuestionario de Percepciones Académicas para la evaluación de las expectativas de los estudiantes de primer año en Enseñanza Superior
} \author{
Fernando Tellado ${ }^{1}$ \\ ${ }^{1}$ Universidad de Vigo \\ ${ }^{2}$ Universidade de Évora \\ ${ }^{3}$ Universidade do Minho \\ ${ }^{4}$ Instituto Superior Politécnico do Porto
}

Manuel Deaño ${ }^{1 *}$, Antonio M. Diniz ${ }^{2}$, Leandro S. Almeida ${ }^{3}$, Sonia Alfonso ${ }^{1}$, Alexandra R. Costa ${ }^{4}$ Mar García-Señorán ${ }^{1}$, Ángeles Conde ${ }^{1}$, Alexandra M. Araujo ${ }^{3}$, Valentín Iglesias-Sarmiento ${ }^{1}$, Paula Gonçalves ${ }^{3}$ y

\begin{abstract}
Resumen: Desde una concepción multidimensional de las expectativas, este artículo pretende analizar la validez y precisión psicométrica de una escala para su medida en los estudiantes universitarios de primer año. La muestra $(N=759)$ estaba compuesta por alumnado de primer año, de diversas titulaciones académicas de la Universidad de Vigo-Campus de Ourense y de la Universidad de Minho. El valor de la $M d n$ de edad fue de 19, siendo sólo el 5.3\% mayor de 23 años. A los participantes se les aplicó un conjunto de 56 ítems agrupados en siete dimensiones diferentes de expectativas. Los resultados del análisis factorial confirmatorio, se obtuvieron con el LISREL. Se garantizó la validez factorial, tanto convergente como discriminante de los factores. Ésta junto con su fiabilidad sugieren que el Cuestionario de Percepciones Académicas quedaría finalmente con 42 ítems distribuidos en siete dimensiones de expectativas: Formación para el empleo/carrera, Desarrollo personal y social, Movilidad estudiantil, Implicación política/ ciudadanía, Presión social, Calidad de formación e Interacción social. Se comprobó la equivalencia del modelo de medida del instrumento en los dos idiomas y en dos grupos resultantes de la bipartición aleatoria de la muestra. Los resultados de la validez estructural de este estudio avalan la utilización del cuestionario para la medida de las expectativas de los estudiantes que inician por primera vez sus estudios en la Enseñanza Superior.

Palabras clave: Expectativas; Enseñanza Superior; estudiantes de primer
\end{abstract} año; ajuste académico; modelos de ecuaciones estructurales.

\section{Introducción}

La Enseñanza Superior (ES) ha sufrido en las últimas décadas, tanto en España como en Portugal, una profunda transformación de su población estudiantil. Frente a una enseñanza dirigida a la élite social y cultural, sirviendo a estudiantes con tradición académica en el seno de sus familias no hace más de medio siglo, en los últimos años, se asiste a una progresiva heterogeneidad de los alumnos que la frecuentan (OECD, 2011a, 2011b). Las necesidades sociales y económicas de mayor formación técnica y cultural de los individuos, entendidas como una mayor expectativa de progresar financiera y socialmente a través de la frecuentación de la ES, de grandes sectores de población, llevaron a su progresiva masificación. La ES en los dos países respondió en los años 70 y 80 a ese incremento exponencial de búsqueda a través de la creación de nuevas instituciones de ES. En ambos países esta respuesta fue realizada a través de la creación o ampliación de la red de ES privada, o en su caso politécnica, y del aumento del número de cursos y de grupos de alumnos

* Dirección para correspondencia [Correspondence address]:

Manuel Deaño Deaño. Departamento de Psicología Evolutiva y Comunicación. Universidad de Vigo. Facultad de Ciencias de la Educación. Campus Ourense. 32004 Ourense (España).

E-mail: deano@uvigo.es
Title: Psychometric properties of the Academic Perceptions Questionnaire for the assessment of first-year university students' expectations.

Abstract: This paper aims to test the psychometric validity and reliability of a measure of first-year university students' expectations, based on a multidimensional conception of expectations. The sample consisted of 759 first-year students, attending various academic degrees at the Universities of Vigo - Ourense and University of Minho. The value $M d n$ age was 19, with only $5.3 \%$ with ages above 23 years. Participants answered a set of 56 items based on seven different dimensions of expectations. Results of confirmatory factor analysis, were carried out with LISREL. Factorial validity, and factors' convergent and discriminant validity were assured. These results, along with evidences reliability, suggest that the Academic Perceptions Questionnaire presents a final structure composed of seven expectation dimensions, including 42 items: Training for employment/career, Personal and social development, Student mobility, Political and citizenship involvement, Social pressure, Quality of education, and Social interaction. The equivalence of measurement model in the two languages and in two groups randomly derived from the full sample was also verified. The results of this structural validity study support the assessment of the expectations of first-year students in Higher Education with the questionnaire.

Key words: Expectations; Higher Education; first-year students; academic adjustment; structural equation modeling.

(Almeida, 2007; Hernández, Sancho, Montané y Sánchez de Serdio, 2011; Magalhães, Amaral y Tavares, 2009). Pero, en los últimos años se ha producido un retroceso en este tipo de demanda, en virtud del impacto de la reducción de las tasas de natalidad en los dos países (Tavares, Tavares, Justino y Amaral, 2008).

Las instituciones de ES y los Gobiernos procuran atraer nuevas poblaciones. Esta apertura se traduce en una nueva fuente de diferenciación de sus públicos. Estudiantes de mayor edad, de clases sociales menos favorecidas, sin tradición familiar de ES, de grupos étnicos minoritarios o con necesidades educativas especiales, entre otros, se acercan hoy a la ES buscando, en el marco de políticas de promoción social y de igualdad de derechos, obtener una formación académica de nivel superior (Bradley, Kish, Krudwig, Williams y Wooden, 2002; Infante-Díaz, 2010; Saavedra, Almeida, Gonçalves y Soares, 2004; Soares, 2003). Se habla, entonces, de nuevos públicos o estudiantes no tradicionales, siendo considerable el número de los que reclaman de las instituciones de ES la atención necesaria hacia sus características y necesidades específicas.

En la actualidad, las instituciones de ES tratan de prestar una mayor atención a sus programas curriculares, a su adecuación a la demanda social y de mercado de trabajo, a los procesos de enseñanza-aprendizaje y evaluación de conoci- 
mientos, a la formación pedagógica de sus docentes y a la calidad de vida en el campus (Fernández-Ortiz, González, Fernández-Losa y Segura, 2010; Pichardo, Berbén, De la Fuente y Justicia, 2007). A su vez, los estudiantes que acceden por primera vez a la ES presentan una gran diversidad de características respecto a su nivel de conocimientos y aptitudes cognitivas (Almeida, Guisande y Paisana, 2012; Deaño, García-Señorán y Sepúlveda, 1997), a sus niveles de motivación (Deaño, Gómez y García-Señorán, 1997) y proyectos vocacionales, a su madurez psicológica, y a su autonomía y bienestar psicológico (Salanova, Martínez, Bresó, LLoréns y Grau, 2005; Soares, Guisande y Almeida, 2007). De entre este conjunto amplio de variables personales de los estudiantes, sus expectativas académicas iniciales son las que centran la atención de este artículo, en la medida en que son una variable importante en su adaptación al contexto universitario (Almeida, et al., 2012; Cole, Kennedy y Ben-Avie, 2009; Howard, 2005; Soares, Almeida y Guisande, 2011).

Las expectativas representan aquello que los estudiantes esperan realizar y concretar en su vida académica, conteniendo bien un componente escolar, bien un componente de integración social. Traducen, así, orientaciones cognitivas y motivacionales realizadas en los contextos de aprendizaje e influencian el nivel de implicación de los estudiantes de ES en su vida escolar y en los desafíos académicos y sociales presentes en esa experiencia (Howard, 2005; Kuh, Gonyea y Williams, 2005). La realización académica y la integración social son componentes integrales del ajuste de los estudiantes a los contextos de la ES (Tinto, 1993), así como dos tareas del desarrollo del joven-adulto universitario (Pascarella y Terenzini, 2005). Por ello, la comprensión de las vivencias de los estudiantes en estos dominios, donde se incluyen sus expectativas, podrá ayudar a la comprensión del fracaso o abandono académico precoz. El estudio de Braxton, Vesper y Hossler (1995) reveló relaciones entre las expectativas de los estudiantes de primer año y su persistencia en la ES, sugiriendo que el nivel de integración académica y social de estos estudiantes está positivamente influenciado por el grado en el que las expectativas para su desarrollo académico e intelectual son correctamente correspondidas.

La investigación muestra que las expectativas y experiencias académicas afectan al nivel de compromiso y de esfuerzo cognitivo, incidiendo en la calidad del ajuste, nivel de aprendizaje, desarrollo psicosocial y persistencia académica (Almeida, Vasconcelos y Mendes, 2008; Igue, Bariani y Milanesi, 2008). También, que las expectativas iniciales de los estudiantes no siempre se concretan. El freshman myth (mito de los estudiantes de primer año) describe unas expectativas demasiado elevadas o fantasiosas para las oportunidades de desarrollo y de aprendizaje asociadas a la ES y que encuentran poco reflejo en sus experiencias reales (Goldfinch y Hughes, 2007; Olsen, et al., 1998). La ilusión inicial puede limitar la percepción de necesidad de adquisición de nuevas competencias por parte de los estudiantes para hacer frente a los desafíos impuestos por la ES (Byrne y Flood, 2005). Gozar de libertad y una mayor autonomía de la familia de origen, establecer relaciones productivas y estimulantes con los profesores, hacer nuevos amigos y establecer relaciones íntimas y acceder a una enseñanza asentada en la investigación, son algunas de las expectativas presentadas por los estudiantes en la ES. Por ello, la confrontación con el primer cuatrimestre lectivo conduce, muchas veces, a una reevaluación y modificación de esas expectativas iniciales (Cook y Leckey, 1999). El desajuste entre expectativas iniciales y la experiencia real de los estudiantes en la ES se traduce en elevados niveles de insatisfacción con la vida académica y la institución de enseñanza, problemas de integración académica y social, así como peores resultados globales en su rendimiento (Baker, McNeil y Siryk, 1985; Braxton, et al., 1995; Howard, 2005).

En síntesis, las expectativas académicas asumen un papel moderador en la implicación y éxito académico de los estudiantes, estando especialmente vinculadas con el ajuste o adaptación a la ES (Baker et al., 1985; Baker y Schultz, 1992; Darlaston-Jones et al., 2003; Jackson, Pancer y Pratt, 2000; Pancer, Hunsberger, Pratt y Alisat, 2000; Smith y Wertlieb, 2005). La investigación en el área diferencia diversos dominios de expectativas, como las relativas al trascurso y éxito en el aprendizaje, a la relaciones con pares y nuevas amistades, a la relación con los profesores, la calidad del campus, de las instalaciones y servicios, o también, al refuerzo de las opciones vocacionales y desarrollo de la carrera. Teniendo en cuenta la literatura en el área y algunos estudios previos, se inició la construcción y validación de un cuestionario sobre las aspiraciones y deseos que los estudiantes tienen al entrar en la Enseñanza Superior, diferenciando perfiles de alumnado en función de sus percepciones sobre lo que este nivel de enseñanza les puede proporcionar (Almeida, Costa, Alves, Gonçalves y Araújo, 2012; Almeida, Guisande, Soares y Saavedra, 2006; Soares y Almeida, 2005; Soares, Guisande, Almeida y Páramo, 2009). Tomando como base las entrevistas al alumnado de $1^{\circ}$ año e investigaciones previas, el cuestionario se fue construyendo y validando en torno a la evaluación de las siguientes dimensiones de expectativas: Formación para el empleo/ carrera, Desarrollo personal y social, Movilidad estudiantil, Implicación política y ciudadanía, Presión social, Calidad de formación e Interacción social.

En este artículo se presentan los resultados de la última fase de construcción y validación de la versión final del Cuestionario de Percepciones Académicas (CPA), en una población de estudiantes universitarios portugueses y españoles. En primer lugar, con la muestra conjunta, se probó la estructura factorial del CPA y después, se examinó su equivalencia de medida en los dos idiomas para comprobar: si los ítems representaban los mismos factores en los dos idiomas; si las cargas factoriales eran invariables en los dos idiomas; y si, los orígenes e intervalos de respuesta a los ítems de las escalas, eran invariables para ambos países. Estando garantizadas estas condiciones a nivel del componente de medida, se pueden estimar diferencias entre grupos en cuanto a su componente estructural: medias, varianzas y covarianzas de los factores (Jöreskog, 2005). Por último, a través de la validez cru- 
zada, se examinó la equivalencia del modelo de medida del CPA en dos grupos resultantes de la bipartición aleatoria de la muestra utilizada en este estudio.

\section{Método}

\section{Participantes}

Para el presente estudio se utilizó una muestra de conveniencia de 759 estudiantes de primer año, de diferentes áreas de formación que ingresaban en la Universidad de Minho ( $n$ =372) y en la Universidad de Vigo-Campus Ourense $(n=$ 387). Su rango de edad se situó entre los 17 y 53 años $(M d n$ = 19; siendo de más de 23 años el 5.3\%). Del total de estudiantes el $62.5 \%$ eran de sexo femenino.

\section{Instrumento}

El Cuestionario de Percepciones Académicas (CPA) traduce las creencias y actitudes que los estudiantes presentan cuando acceden a la ES, cómo se involucran en la ES y esperan que ese contexto satisfaga sus deseos y necesidades. También refleja aquello que los estudiantes consiguen realizar durante el primer año o a lo largo de todos sus estudios.

Para medir las diferentes expectativas que componen el CPA se utilizaron 56 ítems. Estos ítems se distribuyeron en siete dimensiones posibles que los abarcan en su totalidad. La dimensión (i) Formación para el empleo/carrera se refiere a las condiciones de obtención de formación para mejores situaciones de trabajo o de entrada en el mundo laboral, con ítems tales como "Obtener formación para conseguir un buen empleo" o "Tener una preparación necesaria para poder ejercer la profesión que deseo"; (ii) Desarrollo personal y social abarca la autonomía, la autoconfianza y pensamiento crítico, mejora personal a través de las nuevas experiencias de vida académica, con ítems del tipo "Tener nuevas experiencias de vida" o "Ganar confianza en mis potencialidades"; (iii) Movilidad estudiantil, se vincula a la actitud para realizar parte de sus estudios en programas estudiantiles de movilidad internacional, estancias internacionales o trabajos en el extranjero, con ítems como "Participar en programas de intercambio estudiantil universitario (Erasmus, Leonardo, etc.)"; (iv) Implicación política/ciudadanía responde al deseo de poder discutir la vida política, social y económica del país; de comprender cómo poder contribuir a mejorarlas y a la participación en actividades concretas de asociacionismo o voluntariado mediante ítems del tipo de "Participar en grupos de discusión sobre problemas sociales" o "Participar en actividades de voluntariado en la comunidad"; (v) Presión social recoge o agrupa a aquellos ítems referidos al deseo de responder a las expectativas de los padres, colegas y profesores o agradar a personas significativas como "Conseguir corresponder las expectativas de mis familiares" o "Participar en las actividades de estudio para no ser excluido/a por los compañeros/as"; (vi) Calidad de formación se refiere a sentirse desafiado a profundizar en los conocimientos y disponer de los medios personales y materiales que los estimulen comprendiendo ítems como "Profundizar conocimientos/materias en el área de mis estudios" o "Participar en debates o conferencias científicas para profundizar en mis conocimientos en la carrera"; finalmente (vii) Interacción social abarca la voluntad de tener momentos de convivencia y diversión, dedicando a estas actividades un tiempo programado a la semana, distinto del tiempo de estudio y que suponga siempre una relación con sus compañeros con ítems del tipo de "Tener momentos de convivencia y diversión" o "Involucrarme en algún grupo de estudiantes (asociaciones, tunas, etc.)". Para obtener la respuesta a cada uno de los ítems se utilizó una escala de seis puntos desde 1 (total desacuerdo) hasta 6 (total acuerdo).

\section{Procedimiento}

Se realizaron varias reuniones entre los investigadores buscando la equivalencia de constructo y cultural entre la traducción española y la versión portuguesa del cuestionario. También se hicieron entrevistas a estudiantes mediante "reflexión hablada" de los ítems, para certificar que su redacción era perceptible y tenía el significado propuesto.

La recogida de los datos se llevó a cabo dentro de las aulas y en horario académico. Después de la información sobre la naturaleza y objetivos del estudio se solicitó de los estudiantes su participación voluntaria, garantizándoseles la confidencialidad de las respuestas y su uso exclusivo en el marco de la investigación. Los grupos evaluados fueron seleccionados en función de la heterogeneidad de las titulaciones y de la disponibilidad de los profesores. Los ítems fueron presentados, para su aplicación colectiva, por miembros del equipo de investigación con la siguiente instrucción: "Con este cuestionario nos gustaría conocer qué esperas encontrar en este ámbito estudiantil al iniciar por primera vez el curso académico universitario. En él se presentan algunos deseos y aspiraciones que tienen algunos estudiantes universitarios. No se pretende que respondas en función de lo que ya conoces, sino de lo que deseas encontrarte. No existen respuestas correctas o erróneas, lo único importante son tus expectativas. Lee detenidamente cada afirmación y marca en qué medida estás de acuerdo o en desacuerdo con cada una de ellas".

\section{Análisis de datos}

La prueba del modelo oblicuo de siete factores del CPA fue realizada a través del análisis factorial confirmatorio (AFC), utilizando el LISREL 8.53 para Windows (Jöreskog y Sörbom, 2002). Dada la naturaleza ordinal de las respuestas a los ítems se utilizaron estadísticos apropiados de los modelos de ecuaciones estructurales (Bentler y Dudgeon, 1996; Curran, West y Finch, 1996; Flora y Curran, 2004; Jöreskog, 2005). Sabiendo que los datos discretos generan distribuciones no-normales (en efecto, se observó que, de los 56 ítems del CPA, 14 presentaban una $M d n=1.00 ; 26$ una asimetría $\geq 1.25$; y, 34 una curtosis $\geq 1.25$, de los que cuatro tenían 
curtosis $\geq 3.25$ y siete curtosis $\geq 5.25)$ y relaciones nolineales, se hizo necesario un tipo especial de parametrización de los mismos que, en este caso, se realizó con el LISREL 8 (Jöreskog, 2005; Jöreskog y Sörbom, 1993, 1996a). Se examinó en primer lugar, la validez estructural del CPA y después, en una aproximación multigrupo (Cheung y Rensvold, 2002; Jöreskog, 2005; Meredith, 1993), su equivalencia de medida en los dos idiomas y, en una prueba de validez cruzada, en dos grupos resultantes de la bipartición aleatoria de nuestra muestra.

Validez estructural del CPA. En un primer momento, el PRELIS 2 (Jöreskog y Sörbom, 1996a) toma una distribución continua latente de las respuestas a los ítems, segmentada por $m$ - 1 límites distribucionales ( $m=$ número de opciones de respuesta), para estimar una matriz de correlaciones policóricas $(\mathrm{CP})$ de esas respuestas latentes, conjuntamente con la respectiva matriz de covarianza asintótica para ayudar a la estimación. La estimación de CP es teóricamente apropiada para variables ordinales con una distribución bivariada continua latente normal, siendo esta técnica robusta, aún cuando existen violaciones moderadas de esa normalidad (Flora y Curran, 2004). El PRELIS 2 prueba tanto este supuesto como el de la no (multi)colinearidad.

En un segundo momento, las CP sirvieron para la estimación del modelo a través del LISREL 8-SIMPLIS (Jöreskog y Sörbom, 1993), utilizando la técnica de máxima verosimilitud (MV), con la corrección de Satorra-Bentler (1994): esta técnica $\left(\mathrm{MV}_{\mathrm{SB}}\right)$ corrige los errores estándar de las estimaciones y los estadísticos de ajuste de un modelo a los datos empíricos bajo condiciones de no-normalidad multivariada. La técnica $\mathrm{MV}_{\mathrm{SB}}$ presenta un buen desempeño en muestras $N=200$ o mayores, aún en presencia de niveles elevados de no-normalidad multivariada (Curran et al., 1996).

Con este método de aproximación al modelo de ecuaciones estructurales, llamado bivariado latente normal (underlying bivariate normal approach, UBN; Jöreskog y Mustaky, 2001), también sería apropiado recurrir a una técnica de estimación del modelo diferente de la técnica $M V_{\mathrm{SB}}$ : la de los mínimos cuadrados ponderados (full weighted least squares, $W L S)$. Esta técnica exige muestras de gran dimensión para producir estimaciones estables, para que eso suceda, la dimensión mínima que una muestra debe presentar es igual a $(k+1)(k+2) / 2$, donde $k$ es el número de indicadores en el modelo (Jöreskog y Sörbom, 1996b). Así, en este estudio no se utilizó la técnica WLS porque se necesitaría tener, como mínimo, 1653 participantes en la muestra.

Previo al AFC del modelo del CPA por el método $\mathrm{MV}_{\mathrm{SB}^{-}}$ $\mathrm{CP}$, se procedió a su identificación, igualando a 1 la relación de cada uno de los siete factores con uno de los ítems que lo componen (variable de referencia). Para probar la identidad del modelo, es decir, de su ajuste a los datos empíricos, no se utilizó el estadístico $\chi^{2}$, dada su excesiva sensibilidad al tamaño de las muestras (Bentler y Bonett, 1980). En muestras de moderada o gran dimensión, como en el caso de este estudio $(N=759)$, recurrir al test $\chi^{2}$ tendría como consecuen- cia la obtención de un coeficiente muy elevado y estadísticamente significativo, llevando erróneamente a rechazar el modelo (error Tipo I).

El ajuste del modelo a los datos empíricos fue examinado a través de cuatro índices de ajuste práctico (o heurístico). El chi-cuadrado relativo, resultante del cálculo de la razón $\chi^{2} / g l$, debe ser menor o igual que 3 para indicar un buen ajuste (Kline, 2011). Para los otros tres índices, se considera un buen ajuste cuando el comparative fit index (CFI) es próximo o mayor de .95 , el root mean square error of approximation (RMSE $A$ ) es próximo o menor que .06 y el standardized root mean square residual (SRMR) próximo o menor que .08 ( $\mathrm{Hu} y$ Bentler, 1998).

Para asegurar la validez estructural de un modelo, los factores también deben presentar validez convergente (VC), validez discriminante (VD) y fiabilidad (Anderson y Gerbing, 1988). Estas propiedades psicométricas fueron examinadas siguiendo las indicaciones de Fornell y Larcker (1981). La VC fue analizada a través de la varianza media extraída (VME) de los ítems para el respectivo factor, que debe ser igual o superior a .50. La VD resulta de la comparación de la VME con la cantidad de varianza compartida entre los factores $\left(\varphi^{2}=\right.$ cuadrado de la correlación desatenuada), que debe ser inferior a la VME. La fiabilidad de las puntuaciones factoriales (FPF), debe ser, como mínimo, igual o superior a .70 , siendo deseable un valor de .80 para realizar comparaciones entre grupos (Nunnally y Bernstein, 1994).

Equivalencia multigrupo del modelo de medida del CPA. En el test de equivalencia o invarianza del modelo de medida del CPA en los dos idiomas y en dos grupos resultantes de la bipartición aleatoria de la muestra, también se utilizó la aproximación UBN (Jöreskog y Mustaky, 2001). Sin embargo, siguiendo a Jöreskog (2005) y a Millsap y YunTein (2004), se utilizó el PRELIS 2 para fijar a cero y a uno, respectivamente, los dos primeros límites distribucionales de las respuestas latentes de cada grupo de estudiantes de los dos idiomas, o bien, para producir matrices de covarianzas (en lugar de correlaciones) policóricas, conjuntamente con matrices de covarianzas asintóticas. Las estimaciones fueron calculadas para cada grupo bajo límites distribucionales fijados al conjunto de estimaciones de dichos límites de los grupos combinados. Las matrices de covarianzas de este análisis multigrupo sirvieron al test de invarianza del modelo en el LISREL 8.

El examen de invarianza de un modelo comienza, normalmente, por el test de invarianza de su forma a través de los grupos de interés. Con ese modelo, en que todos los parámetros son libremente estimados a través de los grupos, se inicia un proceso de análisis de condiciones de igualdad más restrictivas. Se exploró entonces, la invarianza de la forma del modelo del CPA para comprobar si los dominios cognitivos de los grupos de estudiantes, al compararlos, eran los mismos; su débil invarianza (cargas factoriales iguales) para cotejar si los factores se manifestaban de la misma forma a través de los grupos; y su fuerte invarianza (cargas factoriales, orígenes e intervalos de respuesta a los ítems, iguales) 
para corroborar si la operacionalización de las escalas de medida de los ítems era la misma a través de los grupos (Cheung y Rensvold, 2002; Meredith, 1993).

La invarianza del modelo fue examinada considerando los valores de los índices de ajuste práctico y el valor de la variación del $C F I(\triangle C F I)$ entre un modelo con parámetros libremente estimados y un modelo con ciertos parámetros condicionados a la igualdad a través de los grupos (Cheung y Rensvold, 2002). Un valor de $\Delta C F I$ superior a .01 indica la no invarianza del último modelo (modelo restrictivo).

\section{Resultados}

Validez estructural del CPA. A través del PRELIS 2 se constató tanto la no colinearidad como la normalidad bivariada de las respuestas latentes de los 56 ítems del modelo de siete factores del CPA y, a través del LISREL 8, se comprobó el ajuste aceptable del modelo 1 o M1 (Tabla 1).

Tabla 1. Índices de ajuste práctico del modelo del CPA.

\begin{tabular}{lccccc}
\hline & \multicolumn{5}{c}{ Estimación $\mathrm{MV}_{\mathrm{SB}} \mathrm{CP}$} \\
\cline { 2 - 6 } Modelo & $\chi^{2} / g l$ & Ratio $_{(\chi 2 / g)}$ & CFI & RMSEA & SRMR \\
\hline M1 & $4873.88 / 1463$ & 3.33 & .94 & .06 & .09 \\
M2 & $3350.55 / 1106$ & 3.03 & .95 & .05 & .08 \\
M3 & $2480.85 / 798$ & 3.10 & .95 & .05 & .08 \\
\hline
\end{tabular}

Nota. $\mathrm{MV}_{\mathrm{SB}-\mathrm{CP}}=$ máxima verosimilitud calculado con el Satorra-Bentler, sobre una matriz de correlaciones policóricas. M1 = Modelo 1 de7 factores con 56 ítems; M2 = Modelo 2 de 7 factores con 49 ítems; M3 = Modelo 3 de 7 factores con 42 ítems. CFI $=$ comparative fit index; RMSE $A=$ root mean square error of approximation; $S R M R=$ standardized root mean squared residual.

No obstante, la VME de los factores F5, F6 y F7 fue bastante inferior al valor deseable para el M1 (Tabla 2), indicando problemas de VC en esos factores. En cuanto a la fiabilidad, la FPF de los siete factores fue superior al valor deseable en el M1 (Tabla 2).

También se comprobó la existencia de problemas de VD del M1 entre los pares de factores F2-F4 $\left(\varphi^{2}=.58 ; \mathrm{VME}_{\mathrm{F} 2}=\right.$ .53 y $\left.\mathrm{VME}_{\mathrm{F} 4}=.52\right), \mathrm{F} 2-\mathrm{F} 5\left(\varphi^{2}=.64 ; \mathrm{VME}_{\mathrm{F} 2}=.53\right.$ y VME $\mathrm{V} 5$ $=.40), \mathrm{F} 2-\mathrm{F} 6\left(\varphi^{2}=.58 ; \mathrm{VME}_{\mathrm{F} 2}=.53\right.$ y $\left.\mathrm{VME}_{\mathrm{F} 6}=.40\right)$ y F4$\mathrm{F} 6\left(\varphi^{2}=.62 ; \mathrm{VME}_{\mathrm{F} 4}=.52\right.$ y VME $\left.\mathrm{VM}_{\mathrm{F}}=.40\right)($ Tablas 2 y 3$)$.

Conforme a lo esperado, la exclusión de los ítems del M1 que peor representaban a los factores F5 (ítems 18, 23 y 41), F6 (ítems 3, 22) y F7 (ítems 35 y 47) (i.e., ítems con valores de $R^{2}$ más bajos; Tabla 2) produjo un aumento de las respectivas VME en el modelo 2 o M2 (Tabla 2), mejorando su VC y, también, el ajuste del M2, ahora con 49 ítems (Tabla1). En cuanto a los problemas de VD entre los pares de factores F2-F4, F2-F5, F2-F6 y F4-F6, se produjo un patrón mixto de resultados: disminuyendo entre los pares F2-F5 $\left(\varphi^{2}=.55\right.$; $\mathrm{VME}_{\mathrm{F} 2}=.54$ y VME$\left._{\mathrm{F} 5}=.50\right), \mathrm{F} 2-\mathrm{F} 6\left(\varphi^{2}=.55 ; \mathrm{VME}_{\mathrm{F} 2}=.54\right.$ y $\left.\mathrm{VME}_{\mathrm{F} 6}=.44\right), \mathrm{F} 4-\mathrm{F} 6\left(\varphi^{2}=.61 ; \mathrm{VME}_{\mathrm{F} 4}=.52 \mathrm{y} \mathrm{VME}_{\mathrm{F} 6}=\right.$ $.44)$; y aumentando entre F2-F4 $\left(\varphi^{2}=.61 ; \mathrm{VME}_{\mathrm{F} 2}=.54 \mathrm{y}\right.$ $\left.\mathrm{VME}_{\mathrm{F} 4}=.52\right)($ contrastar M2 con M1 en Tablas 2 y 3$)$.
Tabla 2. Modelo del CPA: Estimaciones factoriales, varianza media extraída y fiabilidad de las puntuaciones factoriales.

\begin{tabular}{|c|c|c|c|c|c|c|}
\hline \multirow[b]{3}{*}{ Ítem (Factor) } & \multicolumn{6}{|c|}{$\mathrm{MV}_{\mathrm{SB}}-\mathrm{PC}$} \\
\hline & \multicolumn{2}{|c|}{ M1 } & \multicolumn{2}{|c|}{ M2 } & \multicolumn{2}{|c|}{ M3 } \\
\hline & $\bar{\beta}$ & $\mathrm{R}^{2}$ & $\beta$ & $R^{2}$ & $\beta$ & $R^{2}$ \\
\hline $1(\mathrm{~F} 1)$ & .64 & .41 & .64 & .41 & .63 & .40 \\
\hline $2(\mathrm{~F} 1)$ & .58 & .34 & .58 & .34 & --- & --- \\
\hline $11(\mathrm{~F} 1)$ & .83 & .69 & .83 & .69 & .83 & .69 \\
\hline 14 (F1) & .84 & .71 & .84 & .71 & .84 & .71 \\
\hline $19(\mathrm{~F} 1)$ & .82 & .67 & .82 & .67 & .82 & .67 \\
\hline 25 (F1) & .84 & .71 & .84 & .71 & .84 & .71 \\
\hline $29(\mathrm{~F} 1)$ & .84 & .71 & .84 & .71 & .84 & .71 \\
\hline 37 (F1) & .83 & .69 & .83 & .69 & .83 & .69 \\
\hline 39 (F1) & .75 & .56 & .75 & .56 & .75 & .56 \\
\hline VME & .61 & & .61 & & .64 & \\
\hline FPF & .93 & & .93 & & 93 & \\
\hline $9(\mathrm{~F} 2)$ & .67 & .45 & .68 & .46 & --- & --- \\
\hline 15 (F2) & .70 & .49 & .70 & .49 & .70 & .49 \\
\hline 27 (F2) & .70 & .49 & .71 & .50 & .71 & .50 \\
\hline 31 (F2) & .78 & .61 & .79 & .62 & .79 & .62 \\
\hline 34 (F2) & .77 & .59 & .77 & .59 & .78 & .61 \\
\hline $42(\mathrm{~F} 2)$ & .70 & .49 & .70 & .49 & .69 & .48 \\
\hline 43 (F2) & .75 & .56 & .75 & .56 & .76 & .58 \\
\hline 48 (F2) & .67 & .45 & .67 & .45 & --- & --- \\
\hline 49 (F2) & .80 & .64 & .80 & .64 & .80 & .64 \\
\hline $50(\mathrm{~F} 2)$ & .74 & .55 & .74 & .55 & .74 & .55 \\
\hline VME & .53 & & .54 & & .56 & \\
\hline FPF & .92 & & .92 & & 91 & \\
\hline $4(\mathrm{~F} 3)$ & .59 & .35 & .59 & .35 & .59 & .35 \\
\hline 7 (F3) & .81 & .66 & .81 & .66 & .81 & .66 \\
\hline 21 (F3) & .86 & .74 & .86 & .74 & .86 & .74 \\
\hline $26(\mathrm{~F} 3)$ & .68 & .46 & .68 & .46 & .68 & .46 \\
\hline 28 (F3) & .80 & .64 & .80 & .64 & .80 & .64 \\
\hline $30(\mathrm{~F} 3)$ & .66 & .44 & .67 & .45 & .67 & .45 \\
\hline 40 (F3) & .85 & .72 & .85 & .72 & .85 & .72 \\
\hline $55(\mathrm{~F} 3)$ & .82 & .67 & .82 & 67 & .82 & .67 \\
\hline VME & .58 & & .59 & & .59 & \\
\hline FPF & .92 & & .92 & & .92 & \\
\hline
\end{tabular}

Con el objetivo de disminuir los problemas de VD del M2 con 49 ítems, se eliminaron los ítems 9 y 48 del F2, el ítem 10 del F4, el ítem 20 del F5 y los ítems 36 y 38 del F6 (items con valores de $R^{2}$ más bajos; Tabla 2 ). También se excluyó el ítem 2 del $F 1$ debido a su valor $\mathrm{R}^{2}$ más bajo comparado con los demás ítems de este factor (Tabla 2). Así la prueba para el nuevo modelo 3 o M3, con 42 ítems, reveló que estaba bien ajustado (Tabla 1) y se pudo comprobar un incremento de la VME de todos los factores, excepto la del F3 que se mantuvo constante (Tabla 2).

De acuerdo a la Tabla 2 para el M3, en este proceso de reespecificación de los factores $\mathrm{F} 1, \mathrm{~F} 2$ y F3 quedaron cada uno con ocho ítems y el F4 con seis, presentando estos cuatro factores valores de VME y de FPF por encima del mínimo exigido. El factor F5 quedó con cuatro ítems, presentando un valor de VME y de FPF también por encima del mínimo exigido. Los factores F6 y F7 quedaron con cuatro ítems también, presentando valores de VME y de FPF por debajo de lo deseable. 
Tabla 2 (continuación). Modelo del CPA: Estimaciones factoriales, varianza media extraída y fiabilidad de las puntuaciones factoriales.

\begin{tabular}{|c|c|c|c|c|c|c|}
\hline \multirow[b]{3}{*}{ Ítem (Factor) } & \multicolumn{6}{|c|}{$\mathrm{MV}_{\mathrm{SB}}-\mathrm{PC}$} \\
\hline & \multicolumn{2}{|c|}{ M1 } & \multicolumn{2}{|c|}{ M2 } & \multicolumn{2}{|r|}{ M3 } \\
\hline & $\bar{\beta}$ & $R^{2}$ & $\beta$ & $R^{2}$ & $\beta$ & $R^{2}$ \\
\hline $10(\mathrm{~F} 4)$ & .57 & .32 & .57 & .32 & --- & --- \\
\hline $12(\mathrm{~F} 4)$ & .75 & .56 & .74 & .55 & .74 & .55 \\
\hline $16(\mathrm{~F} 4)$ & .74 & .55 & .74 & .55 & .74 & .55 \\
\hline 17 (F4) & .77 & .59 & .77 & .59 & .75 & .56 \\
\hline $24(\mathrm{~F} 4)$ & .64 & .41 & .64 & .41 & .64 & .41 \\
\hline $33(\mathrm{~F} 4)$ & .76 & .58 & .77 & .59 & .76 & .58 \\
\hline $52(\mathrm{~F} 4)$ & .78 & 61 & .78 & 61 & .80 & .64 \\
\hline VME & .52 & & .52 & & .55 & \\
\hline FPF & .88 & & .88 & & .88 & \\
\hline $8(\mathrm{~F} 5)$ & .72 & .52 & .75 & .56 & .75 & .56 \\
\hline $18(\mathrm{~F} 5)$ & .49 & .24 & --- & --- & --- & --- \\
\hline $20(\mathrm{~F} 5)$ & .63 & .40 & .62 & .38 & --- & --- \\
\hline $23(\mathrm{~F} 5)$ & .48 & .23 & --- & --- & --- & --- \\
\hline $41(\mathrm{~F} 5)$ & .58 & .34 & -- & --- & --- & --- \\
\hline 44 (F5) & .63 & .40 & .64 & .41 & .58 & .34 \\
\hline 46 (F5) & .79 & .62 & .83 & .67 & .82 & .67 \\
\hline $51(\mathrm{~F} 5)$ & .69 & .48 & .67 & .45 & .71 & .50 \\
\hline VME & .40 & & .50 & & .52 & \\
\hline FPF & 84 & & .83 & & .81 & \\
\hline $3(\mathrm{~F} 6)$ & .53 & .28 & --- & --- & --- & --- \\
\hline $22(\mathrm{~F} 6)$ & .61 & .37 & -- & --- & --- & --- \\
\hline $36(\mathrm{~F} 6)$ & .64 & .41 & .64 & .41 & --- & --- \\
\hline 38 (F6) & .63 & .40 & .64 & .41 & --- & --- \\
\hline 45 (F6) & .64 & .41 & .65 & .42 & .61 & .37 \\
\hline 53 (F6) & .70 & .49 & .70 & .49 & .74 & .55 \\
\hline $54(\mathrm{~F} 6)$ & .64 & .41 & .65 & .42 & .67 & .45 \\
\hline $56(\mathrm{~F} 6)$ & .66 & .44 & 69 & .48 & .70 & .49 \\
\hline $\mathrm{VME}$ & .40 & & .44 & & .46 & \\
\hline FPF & .84 & & .82 & & .78 & \\
\hline $5(\mathrm{~F} 7)$ & .74 & .55 & .79 & .62 & .79 & .62 \\
\hline $6(\mathrm{~F} 7)$ & .72 & .52 & .75 & .56 & .75 & .56 \\
\hline 13 (F7) & .58 & .34 & .60 & .36 & .61 & .37 \\
\hline 32 (F7) & .62 & .38 & .59 & .35 & .59 & .35 \\
\hline 35 (F7) & .55 & .30 & --- & --- & --- & --- \\
\hline $47(\mathrm{~F} 7)$ & .54 & .29 & -- & --- & -- & --- \\
\hline VME & .40 & & .47 & & .48 & \\
\hline FPF & .80 & & .78 & & .78 & \\
\hline
\end{tabular}

Nota. $\mathrm{F} 1$ = Formación para el empleo/carrera; $\mathrm{F} 2=$ Desarrollo personal y social; F3 $=$ Movilidad estudiantil; $\mathrm{F} 4=$ Implicación política y ciudadania $; \mathrm{F} 5=$ Presión social; F6 $=$ Calidad de formación $; \mathrm{F} 7=$ Interacción social . VME $=$ varianza media extraída; FPF $=$ fiabilidad de las puntuaciones factoriales; $\beta=$ peso factorial estandarizado $(\operatorname{con} \phi<.001) ; R^{2}$ (comunalidad) $=1-\varepsilon($ residuo estandarizado). Ver Tabla 1 para otras abreviaturas.

Finalmente, en cuanto a los problemas de VD del M2, se comprobó que las reespecificaciones realizadas en el M3 mejoraron esta propiedad psicométrica (contrastar M3 con M2 en Tablas 2 y 3). La varianza compartida entre F2-F4 disminuyó ligeramente $\left(\varphi^{2}=.59\right)$ y aumentó la VC de esos factores $\left(\mathrm{VME}_{\mathrm{F} 2}=.56\right.$ y $\left.\mathrm{VME}_{\mathrm{F} 4}=.55\right)$, afectando positivamente a su VD, aunque no lo suficiente para ser aceptable. La varianza compartida entre F2-F5 aumentó ligeramente $\left(\varphi^{2}=\right.$ .56), pero el aumento de la $\mathrm{VC}$ de esos factores $\left(\mathrm{VME}_{\mathrm{F} 2}=\right.$ .56 y $\mathrm{VME}_{\mathrm{F} 5}=.52$ ) afectó positivamente a su VD, especialmente debido a la VC del F2. La varianza compartida entre F2-F6 disminuyó ligeramente $\left(\varphi^{2}=.52\right)$ y aumentó la VC de esos factores $\left(\mathrm{VME}_{\mathrm{F} 2}=.56 \mathrm{y} \mathrm{VME}_{\mathrm{F} 6}=.46\right)$, lo que se tradujo en un incremento de su VD, especialmente debido a la VC del F2. Por último, la varianza compartida entre F4-F6 disminuyó ligeramente $\left(\varphi^{2}=.55\right)$ y aumentó la VC de esos factores $\left(\mathrm{VME}_{\mathrm{F} 4}=.55 \mathrm{y} \mathrm{VME} \mathrm{F}_{\mathrm{F} 6}=.46\right)$, afectando positivamente a su VD, especialmente debido a la VC del F4.

Tabla 3. Correlaciones desatenuadas entre los factores del CPA.

\begin{tabular}{|c|c|c|c|c|c|c|c|}
\hline \multirow[b]{2}{*}{ Factor } & \multicolumn{7}{|c|}{ M1; estimaciones $\mathrm{MV}_{\mathrm{SB}}-\mathrm{PC}$} \\
\hline & $\overline{\mathrm{F} 1}$ & $\mathrm{~F} 2$ & F3 & F4 & F5 & F6 & F7 \\
\hline$\overline{\mathrm{F} 1}$ & --- & & & & & & \\
\hline $\mathrm{F} 2$ & .71 & --- & & & & & \\
\hline F3 & .50 & .54 & --- & & & & \\
\hline F4 & .48 & .76 & .45 & --- & & & \\
\hline F5 & .50 & .80 & .47 & .60 & --- & & \\
\hline F6 & .62 & .76 & .53 & .79 & .60 & --- & \\
\hline \multirow[t]{2}{*}{ F7 } & .42 & .65 & .66 & .55 & .60 & .53 & --- \\
\hline & \multicolumn{7}{|c|}{ M2; estimaciones MV $\mathrm{SB}-\mathrm{PC}$} \\
\hline Factor & $\overrightarrow{\mathrm{F} 1}$ & $\mathrm{~F} 2$ & $\mathrm{~F} 3$ & $\mathrm{~F} 4$ & F5 & F6 & F7 \\
\hline $\mathrm{F} 1$ & --- & & & & & & \\
\hline $\mathrm{F} 2$ & .71 & --- & & & & & \\
\hline F3 & .50 & .54 & --- & & & & \\
\hline F4 & .48 & .78 & .45 & --- & & & \\
\hline F5 & .43 & .74 & .43 & .56 & --- & & \\
\hline F6 & .59 & .74 & .49 & .78 & .56 & --- & \\
\hline \multirow[t]{2}{*}{ F7 } & .45 & .61 & .63 & .50 & .49 & .43 & --- \\
\hline & \multicolumn{7}{|c|}{ M3; estimaciones MV $\mathrm{SB}-\mathrm{PC}$} \\
\hline Factor & $\mathrm{F} 1$ & $\mathrm{~F} 2$ & $\mathrm{~F} 3$ & F4 & F5 & F6 & F7 \\
\hline $\mathrm{F} 1$ & -- & & & & & & \\
\hline $\mathrm{F} 2$ & .71 & --- & & & & & \\
\hline $\mathrm{F} 3$ & .51 & .52 & --- & & & & \\
\hline F4 & .48 & .77 & .45 & --- & & & \\
\hline F5 & .47 & .75 & .42 & .56 & --- & & \\
\hline F6 & .60 & .72 & .51 & .74 & .57 & --- & \\
\hline F7 & .45 & .59 & .63 & .49 & .50 & .42 & -- \\
\hline
\end{tabular}

Nota. Todas las correlaciones desatenuadas $(\varphi) \operatorname{con} p<.001$. Ver Tabla 2 para abreviaturas.

Como la mayoría de correlaciones desatenuadas entre los factores del modelo oblicuo del CPA fueron elevadas, especialmente para el M3 (Tabla 3), se podría pensar que la prueba mide, esencialmente, un factor general. Se probaron, entonces, dos modelos alternativos: uno unifactorial, que no estaba bien ajustado $\left(\mathrm{SB} \chi^{2}=8543.06 ; g l=819\right.$; $\mathrm{Ratio}_{(\mathrm{SB} \chi 2 / g l)}$ $=10.43 ;$ CFI $=.89 ; \mathrm{RMSE} A=.11 ;$ SRMR $=.10)$; otro jerárquico, con un factor de segundo orden que subsume los siete factores de primer orden, que presentaba un buen ajuste $\left(\mathrm{SB} \chi^{2}=2612.30 ; g l=812 ; \operatorname{Ratio}_{\left(\mathrm{SB} \chi^{2} / g l\right.}=3.22 ; \mathrm{CFI}=.95\right.$; RMSEA $=.05 ;$ SRMR $=.09)$. Comparando el ajuste de este último modelo con el M3 (Tabla 1), difícilmente se consiguió optar por uno de ellos. Recurriendo al expected cross-validation index (ECVI; Browne y Cudeck, 1993), que sirve para la selección de modelos (adoptando el que tenga un ECVI más bajo), se comprobó que el modelo jerárquico presentaba un valor $(E C V I=3.69)$ superior al del modelo oblicuo (ECVI = 3.55). Así, se rechazó la hipótesis, empíricamente derivada, de que el CPA mide un factor general. 
Equivalencia multigrupo del modelo de medida del CPA. A través del PRELIS 2 se constató, tanto la no colinearidad como la normalidad bivariada de las respuestas latentes de los 42 ítems del modelo de siete factores del CPA en el grupo de estudiantes españoles, en el grupo de estu- diantes portugueses y en los grupos de estudiantes resultantes de la bipartición aleatoria de la muestra.

Los resultados obtenidos con el LISREL 8 para el test de invarianza del modelo de medida del CPA a través de los grupos de estudiantes españoles y portugueses son los recogidos en la Tabla 4.

Tabla 4. Invarianza del modelo de medida del CPA en los dos idiomas.

\begin{tabular}{|c|c|c|c|c|c|c|c|}
\hline \multirow[b]{3}{*}{ Modelo } & \multirow[b]{3}{*}{$\chi^{2} / g l$} & \multicolumn{6}{|c|}{ Estimación $\mathrm{MV}_{\mathrm{SB}}-\mathrm{CovP}$} \\
\hline & & \multirow[b]{2}{*}{$\operatorname{Ratio}_{\left(\chi^{2} / g l\right)}$} & \multirow[b]{2}{*}{ RMSEA } & \multicolumn{2}{|c|}{ SRMR } & \multirow[b]{2}{*}{ CFI } & \multirow[b]{2}{*}{$\triangle C F I$} \\
\hline & & & & Espanhol & Português & & \\
\hline$\overline{\mathrm{M} 1}$ & $3214.71 / 1596$ & 2.01 & .05 & .09 & .08 & .982 & -- \\
\hline M2 & $3313.25 / 1631$ & 2.03 & .05 & .09 & .10 & .982 & .000 \\
\hline M3 & $3603.62 / 1673$ & 2.15 & .06 & .09 & .10 & .979 & .003 \\
\hline
\end{tabular}

Nota. $\mathrm{MV}_{\mathrm{SB}}-\mathrm{CovP}=$ máxima verosimilitud calculado con el Satorra-Bentler, sobre matrices de covarianzas policóricas. M1 = invarianza de forma; M2 = invarianza débil; M3 = invarianza fuerte. $\Delta=$ variación. Ver Tabla 1 para otras abreviaturas.

Como se puede ver en la Tabla 4, los valores de los índices de ajuste práctico indicaron la invarianza débil y fuerte del modelo de medida del CPA a través de los grupos, aunque con resultados ligeramente elevados para el $S R M R$, a saber, en el grupo de portugueses en M2 y M3. También los valores obtenidos para la variación del CFI apuntaron a esa invarianza $(\triangle C F I<.01)$.
Los resultados del examen de validez cruzada del modelo de medida del CPA, obtenidos a través del test de invarianza en los dos grupos resultantes de la bipartición aleatoria de la muestra (Grupo 1, $n=380$; Grupo 2, $n=379$ ), se presentan en la Tabla 5.

Tabla 5. Invarianza del modelo de medida del CPA: validez cruzada

\begin{tabular}{|c|c|c|c|c|c|c|c|}
\hline \multirow[b]{3}{*}{ Modelo } & \multirow[b]{3}{*}{$\chi^{2 / g l}$} & \multicolumn{6}{|c|}{ Estimación $\mathrm{MV}_{\mathrm{SB}}-\mathrm{CovP}$} \\
\hline & & \multirow[b]{2}{*}{$\operatorname{Ratio}_{(\chi 2 / g l}$} & \multirow[b]{2}{*}{ RMSEA } & \multicolumn{2}{|c|}{$S R M R$} & \multirow[b]{2}{*}{ CFI } & \multirow[b]{2}{*}{$\triangle C F I$} \\
\hline & & & & Grupo 1 & Grupo 2 & & \\
\hline$\overline{\mathrm{M} 1}$ & $3138.38 / 1596$ & 1.97 & .05 & .09 & .09 & .983 & - \\
\hline M2 & $3290.53 / 1631$ & 2.02 & .05 & .09 & .09 & .982 & .001 \\
\hline M3 & $3418.28 / 1673$ & 2.04 & .05 & .09 & .09 & .981 & .002 \\
\hline
\end{tabular}

En la Tabla 5 se puede comprobar que la validez cruzada del modelo fue asegurada: el modelo estaba bien ajustado y presentaba invarianza débil y fuerte a través de los grupos.

\section{Discusión y conclusiones}

La investigación muestra que las expectativas iniciales de los estudiantes al ingresar en la Universidad afectan al nivel de compromiso y de esfuerzo cognitivo, incidiendo en la calidad del ajuste, nivel de aprendizaje, desarrollo psicosocial y persistencia académica (Almeida, et al., 2008; Igue, et al., 2008; Pascarella y Terenzini, 2005; Tinto, 1993). La aproximación multidimensional permite conceptualizar las expectativas como las experiencias que se van produciendo en la vida académica y configurando su ajuste a ella, favoreciendo de este modo la adaptación a la ES (Howard, 2005; Kuh, et al., 2005).

Los resultados de este trabajo de investigación identifican, mediante el Cuestionario de Percepciones Académicas (CPA), los deseos y aspiraciones que los estudiantes españoles y portugueses presentan cuando acceden a la ES, cómo pretenden desenvolverse en ella y cómo esperan que este contexto los satisfaga. Estas aspiraciones conforman las expec- tativas que favorecen la realización académica y la integración social. Los componentes de realización académica estarían representados en el cuestionario por las dimensiones de Formación para el empleo/carrera, Movilidad estudiantil y Calidad de formación. Los componentes de integración social están medidos por las dimensiones de Interacción social, Presión social, Implicación política/ ciudadanía y Desarrollo personal y social.

El CPA evalúa siete tipos diferentes de expectativas: F1, Formación empleo/carrera; F2, Desarrollo personal y social; F3, Movilidad estudiantil; F4, Implicación politica/ ciudadanía; F5, Presión social; F6, Calidad de formación; y, F7, Interacción social. El proceso de validación del modelo operacionalizado por la prueba realizada a través del AFC mostró que presenta un buen ajuste a los datos empíricos. Como resultado de ese proceso los factores F1, F2, F3 (todos con ocho ítems), F4 (con seis ítems) y F5 (con cuatro ítems) presentaron coeficientes de validez convergente y de fiabilidad por encima de los límites mínimos exigidos. Los factores F6 y F7 (ambos con cuatro ítems) presentaron valores inferiores a los deseables psicométricamente. Se produjeron algunas dificultades en la validez discriminante entre el F2 (Desarrollo personal y social) y los factores F4 (Implicación política/ciudadanía), F5 (Presión social) y F6 (Calidad de formación), así como entre el F4 y F6. 
Una interpretación es que estas dificultades parecen reflejar una proximidad de contenido entre los ítems asociados al desarrollo psicosocial con los relacionados con Implicación politica/ ciudadania, Presión social y Calidad de formación. La proximidad encontrada entre los ítems de la Implicación politica/ ciudadanía y la Calidad de formación podría, a su vez, traducir un mayor grado de involucración por parte de los estudiantes en tareas académicas de calidad superior (por ejemplo participar en debates o en proyectos de investigación de sus profesores).

Aunque con algunas limitaciones, los indicadores psicométricos de fiabilidad y validez obtenidos, son satisfactorios para la utilización del CPA en la medida de las expectativas de los estudiantes de ES españoles y portugueses que inician por primera vez sus estudios en este ámbito académico. Sería conveniente la consideración de la diferencia deseable entre el contenido ampliado de los factores F4, F5, F6, F7 y el de los ítems que permanecen en el F2, así como de la diferenciación del aporte de nuevos ítems del F4 y del F6. Es posible también que, al menos para los estudiantes de primer año, los ítems de desarrollo psicosocial (F2) se puedan entender como transversales a las dimensiones de expectativas Implicación politica/ciudadanía, Presión social y Calidad de forma-

\section{Referencias}

Almeida, L. S. (2007). Transição, adaptação académica e êxito escolar no ensino superior. Revista Galego-Portuguesa de Psicoloxía e Educación, 14(2), 203-215.

Almeida, L. S., Costa, A. R., Alves, F., Gonçalves, P. y Araújo, A. (2012). Expetativas académicas dos alunos do ensino superior: construção e validação de uma escala de avaliação. Psicologia, Educação e Cultura, XVI(I), 70-85.

Almeida, L. S., Guisande, M. A. y Paisana, J. (2012). Participación extracurricular, ajuste y rendimiento académico en la Enseñanza Superior: Un estudio con estudiantes portugueses. Anales de Psicología, 28(3), 860-865.

Almeida, L. S., Guisande, M. A., Soares, A. P. y Saavedra, L. (2006). Acesso e sucesso no Ensino Superior em Portugal: Questões de género, origem sóciocultural e percurso académico dos alunos. Psicologia: Reflexão e Crítica, 19(3), 507-514.

Almeida, L., Vasconcelos, R. y Mendes, T. (2008). O abandono dos estudantes no Ensino Superior: um estudo na Universidade do Minho. Revista GalegoPortuguesa de Psicoloxía e Educación, 16(1,2), 111-119.

Anderson, J. C. y Gerbing, D. W. (1988). Structural equation modeling in practice: A review and recommended two step approach. Psychological Bulletin, 103(3), 411-423.

Bagozzi, R. P. y Yi, I. (1998). On the evaluation of structural equation models. Journal of the Academy of Marketing Science, 16(1), 74-94.

Baker, R. W., McNeil, O. V. y Siryk, B. (1985). Expectations and reality in freshman adjustment to college. Journal of Counselling Psychology, 32(1), 94-103.

Baker, R. W. y Schultz, K. L. (1992). Measuring expectations about college adjustment. NACADA Journal, 12(2), 23-32.

Bentler, P. M. y Bonett, D. G. (1980). Significance tests and goodness of fit in the analysis of covariance structures. Psychological Bulletin, 88(3), 588-606.

Bentler, P. M. y Dudgeon, P. (1996). Covariance structure analysis: Statistical practice, theory, and directions. Annual Review of Psychology, 47, 563-592.

Bradley, C., Kish, K. A., Krudwig, A. M., Williams, T. y Wooden, O. S. (2002). Predicting faculty-student interaction: An analysis of new student expectations. Journal of Indiana University Student Personnel Association, 2, 72-85.

Braxton, J. M., Vesper, N. y Hossler, D. (1995). Expectations for college and student persistence. Research in Higher Education, 36(5), 595-611.

Browne, M. W. y Cudeck, R. (1993). Alternative ways of assessing model fit. En K. A. Bollen y J. S. Long (Eds.), Testing structural equation models (pp. 136-162). London: SAGE.

Byrne, M. y Flood, B. (2005). A study of accounting students' motives, expectations and preparedness for Higher Education. Journal of Further and Higher Education, 29(2), 111-124. ción. En ese caso, futuras investigaciones deberían ratificar la necesidad efectiva de mantener esta dimensión autónoma en el cuestionario.

Para finalizar, a pesar de las sugerencias de mejora realizadas en cuanto a validez estructural del CPA y a la invarianza del modelo de medida del cuestionario en estudiantes españoles y portugueses (en los dos idiomas), así como en los dos grupos seleccionados aleatoriamente a partir de la muestra utilizada en este estudio, éstas deben considerarse de forma prudente. Atendiendo al proceso no probabilístico de muestreo utilizado en este estudio, sería deseable, en una estrategia de generalización de la validez (Bagozzi y Yi, 1998), proceder a replicarla en otras muestras de población de estudiantes universitarios. Ocurre que, a pesar de estar fuera del alcance de este trabajo, el examen de validez nomológica (Cronbach y Meehl, 1955) del modelo del CPA al nivel del componente de medida y al nivel del componente estructural, también contribuirán a una mayor garantía de validez de la prueba: por ejemplo, examinando su invarianza a través de grupos de estudiantes de sexo femenino y masculino o, a través de grupos de estudiantes de los ámbitos científico, tecnológico, humanístico y social.

Cole, J. S., Kennedy, M. y Ben-Avie, M. (2009). The role of precollege data in assessing and understanding student engagement in college. New Directions for Institutional Research, 141, 55-69.

Cook, A. y Leckey, J. (1999). Do expectations meet reality? A survey of changes in first year student opinion. Journal of Further and Higher Education, 23(2), 157-171.

Cronbach, L. J. y Meehl, P. E. (1955). Construct validity in psychological tests. Psychological Bulletin, 52(4), 281-302.

Curran, P. J., West, S. G. y Finch, J. F. (1996). The robustness of test statistics to nonnormality and specification error in confirmatory factor analysis. Psychological Methods, 1(1), 16-29.

Cheung, G.W. y Rensvold, R. B. (2002). Evaluating goodness-of-fit indexes for testing measurement invariance. Structural Equation Modeling, 9(2), 233-255.

Darlaston-Jones, D., Pike, L., Cohen, L., Young, A., Haunold, S. y Drew, N. (2003). Are they being served? Student expectations of higher education. Issues In Educational Research, 13(1), 31-52.

Deaño, M., García-Señorán, M. y Sepúlveda, J. (1997). Factores de éxito en alumnos de último curso de carrera en la Universidad de Vigo. Caderno de Innovación i Educación (CIE), 1, 203-238.

Deaño, M., Gómez, J. y García-Señorán, M. (1997). Perfil del alumnado de la Universidad de Vigo a partir de la evaluación docente del profesorado. Caderno de Innovación $i$ Educación (CIE), 1, 253-264.

Fernández-Ortiz, R., González, L., Fernández-Losa, N. y Segura, M. J. (2010). Calidad universitaria: expectativas de los estudiantes recién incorporados. Revista Nacional de Administración, 1(2), 17-30.

Flora, D. B. y Curran, P. J. (2004). An empirical evaluation of alternative methods of estimation for confirmatory factor analysis with ordinal data. Psychological Methods, 9(4), 466-491.

Fornell, C. y Larcker, D. F. (1981). Evaluating structural equation models with unobservable variables and measurement error. Journal of Marketing Research, 18(1), 39-50.

Goldfinch, J. y Hughes, M. (2007). Skills, learning styles and success of first-year undergraduates. Active Learning in Higher Education, 8(3), 259-273.

Hernández, F., Sancho, J. M., Montané, A. y Sánchez de Serdio, A. (2011). ¿Cómo los académicos españoles se enfrentan con el cambio? Respuesta desde una investigación de historias de vida profesionales. Barcelona: Universidad de Barcelona. Recuperado de http://hdl.handle.net/2445/20983.

Howard, J. A. (2005). Why should we care about student expectations? En T. E. Miller, B. E. Bender, J. H. Schuh y Associates (Eds.), Promoting reasonable ex- 
pectations: Aligning student and institutional views of the college experience (pp. 10-33). San Francisco, CA: Jossey-Bass.

Hu, L. y Bentler, P. M. (1998). Fit indices in covariance structure modeling: Sensitivity to underparametrized model misspecification. Psychological Methods, 3(4), 424-453.

Igue, E. A., Bariani, I. C. y Milanesi, P. V. (2008). Vivência acadêmica e expectativas de universitários igressantes e concluintes. Psico-USF, 13(2), 155-164.

Infante-Díaz, J. (2010). La reforma de los planes de estudio universitarios de la España democrática. Revista de Educación, 351, 259-282.

Jackson, L. M., Pancer, S. M. y Pratt, M. W. (2000). Great expectations: The relation between expectancies and adjustment during the transition to university. Journal of Applied Social Psychology, 30(10), 2100-2025.

Jöreskog, K. G. (2005). Structural equation modeling with ordinal variables using LISREL. Recuperado http://www.ssicentral.com/lisrel/techdocs/ordinal.pdf

Jöreskog, K. G. y Mustaky, I. (2001). Factor analysis of ordinal variables: A comparison of three approaches. Multivariate Behavioral Research, 36, 347-387.

Jöreskog, K. G. y Sörbom, D. (1993). LISREL 8: Structural equation modeling with the SIMPLIS command language. Chicago, IL: Scientific Software International.

Jöreskog, K. G. y Sörbom, D. (1996a). PRELIS 2: User's reference guide. Chicago, IL: Scientific Software International.

Jöreskog, K. G. y Sörbom, D. (1996b). LISREL 8: User's reference guide. Chicago, IL: Scientific Software International.

Jöreskog, K. G. y Sörbom, D. (2002). LISREL 8.53 for Windows [Computer software]. Chicago, IL: Scientific Software International.

Kline, R. B. (2011). Principles and practice of structural equation modeling ( $3^{\mathrm{a}}$ ed.). New York, NY: Guilford Press.

Kuh, G. D., Gonyea, R. M. y Williams, J. M. (2005). What students expect from college and what they get. En T. E. Miller, B. E. Bender, J. H. Schuch y Associates (Eds.), Promoting reasonable expectations: Aligning student and institutional views of the college experience (pp. 34-64). San Francisco, CA: Jossey-Bass.

Magalhães, A., Amaral, A. y Tavares, O. (2009). Equity, access and institutional competition. Tertiary Education and Management, 15(1), 35-48.

Meredith, W. (1993). Measurement invariance, factor analysis and factorial invariance. Psychometrika, 58(4), 525-543.

Millsap, R. E. y Yun-Tein, J. (2004). Assessing factorial invariance in orderedcategorical measures. Multivariate Behavioral Research, 39(3), 479-515.

Nunnally, J. C. y Bernstein, I. H. (1994). Psychometric theory ( $3^{\mathrm{a}}$ ed.). New York, NY: McGraw-Hill.

OECD (2011a). Education at a Glance 2011: Highlights. OECD Publishing. Recuperado http://www.oecd.org/edu/highereducationandadultlearning/48631550.pdf

OECD (2011b). Education at a Glance 2011: OECD indicators. OECD Publishing. Recuperado

http://www.oecd.org/education/highereducationandadultlearning/4863158 2.pdf
Olsen, D., Kuh, G. D., Schilling, K. M., Schilling, K., Connolly, M., Simmons, A. y Vesper, N. (November, 1998). Great Expectations: What First-Year Students Say They Will Do and What They Actually Do. Paper presented at the Annual Meeting of the Association for the Study of Higher Education, Miami, FL.

Pancer, S. M., Hunsberger, B., Pratt, M. W. y Alisat, S. (2000). Cognitive complexity of expectations and adjustment to university in first year. Journal of Adolescent Research, 15(1), 38-57.

Pascarella, E .T. y Terenzini, P. T. (2005). How College Affects Students: A Third Decade of Research. San Francisco: Jossey-Bass.

Pichardo, M. C., Berbén, A. B. G., De la Fuente, J. y Justicia, F. (2007). El estudio de las expectativas en la universidad: análisis de trabajos empíricos y futuras líneas de investigación. Revista Electrónica de Investigación Educativa, 9(1). Recuperado de http://redie.uabc.mx/vol9no1/contenido-pichardo.htm

Saavedra, L., Almeida, L. S., Gonçalves, A. y Soares, A. P. (2004). Pontos de partida, pontos de chegada: Impacto de variáveis sócio-culturais no ingresso ao Ensino Superior. Sociedade e Cultura 6, Cadernos do Noroeste, 22(1-2), 63-84.

Salanova, M., Martínez, I. M., Bresó, E., LLorens, S. y Grau, R. (2005). Bienestar psicológico en estudiantes universitarios: facilitadores y obstaculizadores del desempeño académico. Anales de Psicología, 21(1), 170-180.

Satorra, A. y Bentler, P. M. (1994). Corrections to test statistics and standard errors in covariance structure analysis. En A. Von Eye y C. C. Clogg (Eds.), Latent variable analysis (pp. 399-419). Thousand Oaks, CA: SAGE.

Smith, J. S. y Wertlieb, E. C. (2005). Do first-year college students' expectations align with their first-year experiences? NASPA Journal, 42(2), 153-174.

Soares, A. P. (2003). Transição e adaptação ao Ensino Superior: Construção e validação de um modelo multidimensional de ajustamento de jovens ao contexto universitário. Unpublished doctoral thesis. Braga: Universidade do Minho.

Soares, A. P. C. y Almeida, L. S. (2005). Questionário de Envolvimento Académico (QEA): Novos elementos para a sua validação. Psicología: Teoria, Investigação e Prática, 10(2), 139-158.

Soares, A. P., Almeida, L. S. y Guisande, M. A. (2011). Ambiente académico y adaptación a la universidad: Un estudio con estudiantes de $1^{\circ}$ año de la Universidad do Minho. Revista Iberoamericana de Psicología y Salud, 2(1), 99-121.

Soares, A. P., Guisande, M. A. y Almeida, L. S. (2007). Autonomía y ajuste académico: Un estudio con estudiantes portugueses de primer año. International Journal of Clinical and Health Psychology, 7(3), 753-765.

Soares, A. P., Guisande, M. A., Almeida, L. S. y Páramo, M. F. (2009). Academic achievement in first-year Portuguese college students: The role of academic preparation and learning strategies. International Journal of Psychology, 44(3), 204-212.

Tavares, D., Tavares, O., Justino. E. y Amaral, A. (2008). Students' preferences and needs in Portuguese Higher Education. European Journal of Education, 43(1), 107-122.

Tinto, V. (1993). Leaving college: Retbinking the causes and cures of student attrition ( $2^{\mathrm{a}}$ ed.). Chicago, IL: University of Chicago Press.

(Articulo recibido: 26-10-2012; revisión recibida: 12-2-2013; aceptado: 21-6-2013) 


\section{Apéndice}

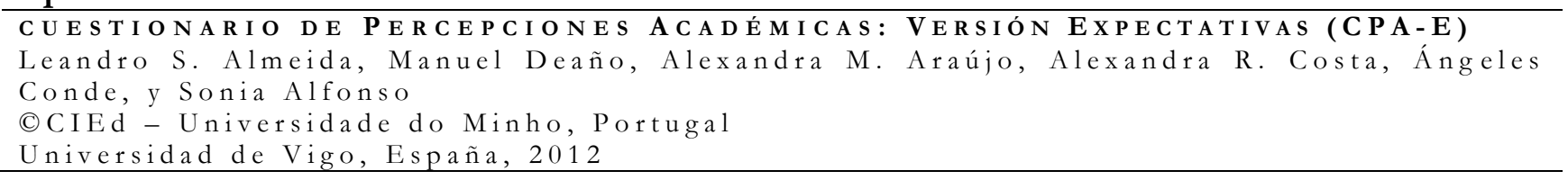

"Lee detenidamente cada afirmación y marca en qué medida estás de acuerdo o en desacuerdo con cada una de ellas"

1. Conseguir una profesión de prestigio.

4. Sentirme en una universidad que se esfuerza por internacionalizarse.

5. Tener momentos de convivencia y diversión.

6. Practicar alguna actividad extracurricular (deporte, cultura, otros).

7. Participar en programas de intercambio estudiantil universitario: Erasmus, Leonardo, etc...

8. Conseguir corresponder a las expectativas de mis familiares.

11. Tener mejores salidas profesionales en el mercado de trabajo.

12. Comprender cómo puedo contribuir a mejorar el mundo y la sociedad.

13. Tener un horario semanal que me permita realizar otras actividades más allá del estudio.

14. Aumentar la posibilidad de conseguir un empleo estable en el futuro.

15. Aprovechar las oportunidades académicas para mejorar mi identidad, autonomía, autoconfianza, etc...

16. Involucrarme en la resolución de problemas de las personas menos favorecidas.

17. Tener una visión crítica del mundo y pensar en cómo transformarlo.

19. Obtener formación para conseguir un buen empleo.

21. Conseguir realizar alguna estadía en otro país.

24. Participar en actividades de voluntariado en la comunidad.

25. Capacitarme para obtener éxito profesional en el futuro.

26. Sentir que estoy en una Universidad que favorece la movilidad estudiantil.

27. Desarrollar mis características de personalidad.

28. Obtener formación que me permita ampliar los horizontes de empleo en el extranjero.

29. Asegurar una carrera profesional satisfactoria al terminar los estudios.

30. Obtener formación de calidad internacional.

31. Ganar confianza en mis potencialidades.

32. Participar regularmente en fiestas estudiantiles universitarias.

33. Formarme como ciudadano comprometido con los desafíos de la sociedad actual.

34. Tener objetivos en la vida y saber hacia dónde "quiero ir".

37. Tener la preparación necesaria para poder ejercer la profesión que deseo.

39. Conseguir prácticas que faciliten la entrada en el mercado de trabajo.

40. Participar en la movilidad estudiantil pasando algún tiempo de mis estudios en otro país.

42. Tener amigos que me ayuden a superar posibles dificultades.

43. Aprender a manejarse autónomamente con las dificultades de la vida.

44. No quedar atrás de otros compañeros en las notas o calificaciones.

45. Participar en debates o conferencias científicas para profundizar mis conocimientos en la carrera.

46. No decepcionar a familia o amigos en el rendimiento académico.

49. Adquirir competencias para convertirme en un adulto más responsable y autónomo.

50. Tener nuevas experiencias de vida.

51. Aprovechar la oportunidad de formación que mi familia me asegura.

52. Contribuir a la mejora de la condición humana o en el bienestar de las personas.

53. Profundizar conocimientos/materias en el área de mis estudios.

54. Conseguir participar en proyectos de investigación de los profesores de mi facultad.

55. Estar dispuesto/a a realizar estadías en el extranjero para obtener un título reconocido internacionalmente.

56. Tener un buen rendimiento académico para corresponder a la inversión que la sociedad hace en mi formació superior. 